\title{
Non-linear modelling of the effects of strain on transition metal surfaces
}

I. G. Shuttleworth,

School of Science and Technology,

Nottingham Trent University,

Nottingham NG11 8NS.

UK

E-mail: $\underline{\text { ian.shuttleworth@ntu.ac.uk }}$

\begin{abstract}
A sequence of polynomial expressions have been shown to describe the strained surface energy of low-index hexagonal and square transition metal surfaces. Distinguishable functions describe the hexagonal $\mathrm{FCC}(111)$ and $\mathrm{HCP}(0001)$ surfaces, but a single function describes the FCC(100) and BCC(100) surfaces. A far weaker dependence exists between the strained surface energy and the electronic state of the surface, and the competition between geometric and electronic states across is discussed.
\end{abstract}

\section{Keywords}

Surface energy; transition metal; d-band effects; strain engineering; non-linear elasticity.

\section{Research highlights}

- The behaviour of transition metal surface energy under strain is predominantly geometry dependant

- A simple, cubic-order polynomial description of this surface energy under strain has been achieved

- Polynomial descriptions can be broadly classified into hexagonal and square functions. Further subdivision based on selvedge structure is then possible.

- The effect of the electronic structure of the surface on the strained surface energy is comparatively weak 


\section{Introduction}

Surface energy is central quantity in the study of metals. The energy arises during cleavage of the bulk metal into two parts [1-2] whereupon the atoms relax from their ideally bulk terminated positions to lower energy sites. The reduction of energy is due to loss of periodicity; surface atoms do not have the same perfectly periodic surroundings that their counterpart atoms in the bulk have, and consequently experience an imbalance in the forces that act on them. This imbalance is relieved by relaxation of the atoms in the surface, and selvedge, regions.

In these circumstances, the underlying bulk atoms remain in equilibrium, and un-stressed and comparison of the surface energies across the d-period show a volcano-type dependence [3]. Contemporary studies [4] of the closely related quantity of surface stress have highlighted the complexity of the field and have reviewed the various models of surface electronic structure that commonly invoked to describe surface relaxation. A central them to these models is the interplay between delocalised $\mathrm{sp}$ and localised $\mathrm{d}$ orbitals, pioneered by Pettifor [5]. The energetic importance of the d-states has been underlined by the more recent Friedel stress model [6] which has successfully modelled the surface stress across systems where the bulk atoms are unstrained.

Understanding surface energy and stress and their response to changes in the bulk is central to both fundamental surface science and it's applications. Contemporary studies of the surface [7] and bulk [8] hydrogen in strained metallic systems have demonstrated that both the binding position and the electronic state of this catalytically-important element depends sensitively on the development of the surface and bulk energy. A number of approaches have been used to calculate surface energy [9-13]. Simulations based on the jellium model [14] have been applied to systems where the underlying bulk atoms are strained and have proposed a parameterisation of the deformed Wigner-Seitz cell. More recent studies have investigated the energies of hexagonal surfaces [15] using density functional theory (DFT) and have identified correlations between the work function and crystallographic orientations of the surfaces. The requirement for DFT level precision in modelling strained surfaces has been further underlined in studies of $\mathrm{Al}, \mathrm{Pd}, \mathrm{Pt}, \mathrm{Au}$ and $\mathrm{Ti}$ [16], the low index (111), (110) and (001) faces of $\mathrm{AlCu}_{3}$ [17] and on transition metal carbide films [18].

The current work will be based on density-functional theory (DFT) level simulations and will survey a range of both square and hexagonal transition metal surfaces. To reduce the model the formalism of non-linear elasticity theory will be adopted [19], an approach which has successfully applied to bulk $\mathrm{MgO}$ [20]. Elasticity theory describes the response of a system to a finite deformation. The formalism is therefore entirely consistent with the type of investigation performed in the current study where the metallic systems will be strained and the response of the surface will be scrutinised. The theory requires that energy of the system is expanded in a Taylor-series of terms in strain, the first order term then describing the linear response and the higher order terms describing non-linear components.

The current work is divided into three sections: in the first section, an outline of the DFT method and the definition of strain used in the current work is presented. The subsequent section presents the surface energy and non-linear response functions, together with their clear 
definition and a discussion of the geometric and electronic issues arising in these fits, and the paper finishes with a conclusions section.

\section{Theory}

Investigations performed in this current work were of the $\mathrm{Fe}, \mathrm{Co}, \mathrm{Ni}, \mathrm{Cu}, \mathrm{Ru}, \mathrm{Rh}, \mathrm{Pd}, \mathrm{Ag}, \mathrm{Os}$, Ir, Pt and Au systems. The structures investigated are summarised in fig. 1. For the FCC systems - Ni, Cu, Rh, Pd, Ag, Ir, Pt and $\mathrm{Au}$ - the hexagonal (111) and square (100) surfaces were simulated, and the hexagonal HCP (0001) and square BCC (100) surfaces were simulated for the HCP - Co, Ru, Os - and BCC - Fe - systems, respectively. The surfaces were strained uniformly in the surface-parallel direction by an amount $\sigma$.

The plane-wave density functional theory (DFT) simulations presented in this work were performed using the Quantum Espresso package [21]. Brillouin zone sampling was performed on a $(6 \times 6 \times 1)$ grid using a first-order Methfessel-Paxton smearing of $0.02 \mathrm{Ry}$ [22]. The Fe, Co and $\mathrm{Ni}$ simulations for spin-polarised whereas all other simulations were not. Norm-conserving pseudopotentials were used for the $\mathrm{Cu}, \mathrm{Ru}, \mathrm{Rh}, \mathrm{Pd}, \mathrm{Os}$, Ir or Pt simulations [23] and ultrasoft pseudopotentials [23, 24] were used for the $\mathrm{Fe}, \mathrm{Co}, \mathrm{Ni}, \mathrm{Ag}$ and $\mathrm{Au}$ simulations [23, 24]. A wave-function kinetic energy cut-off of 50-100 Ry was used for all simulations and a charge density/potential kinetic energy cut-off of $4 \times$ that amount was used for the simulations that used norm-conserving pseudopotentials, and $12 \times$ that amount for the simulations that used ultra-soft pseudopotentials. Table 1 summarises the equilibrium bulk lattice constants determined computationally in the current work $\left(\mathrm{a}_{\mathrm{theo}}\right)$ and experimentally $\left(\mathrm{a}_{\mathrm{expt}}\right)$. For this part of the investigation the Brillouin zone sampling was performed on a $(6 \times 6 \times 6)$ grid.

All investigations were performed using $(1 \times 1)$ surface unit cells and 7 layer slabs, separated by a vacuum of approximately 12 atomic layer spacings. The amount of lattice strain $\sigma$ was defined as

$$
\frac{\mathrm{L}}{\mathrm{L}_{0}}=1+\frac{\sigma(\%)}{100}
$$

$\mathrm{L}$ and $\mathrm{L}_{0}$ are the strained and un-strained lattice constants, respectively. Throughout the current work, strain $\sigma$ was numerically treated as a percentage and $\sigma \in[-5 \%, \ldots,+5 \%]$. During relaxation only the central layer of metal atoms were constrained. The remaining atoms were allowed to relax freely, though their in-plane spacing changed sympathetically with that of the central atomic layer. Because of the small unit cell size the possibility of in-plane reconstructions was not investigated; however, significant reconstruction effects were not anticipated. This is because within the group of metals investigated, few would be expected to reconstruct under the range of strains used. The notable exception to this is Au though that only undergoes reconstruction for large surface unit cells. 


\section{Results and Discussion}

Fig. 2 summarises the surface energies $E_{S}$ which were defined by

$$
\mathrm{E}_{\mathrm{S}}=\frac{1}{2 \mathrm{~A}}\left(\mathrm{E}_{\text {Slab }}-\mathrm{N}_{\text {Slab }} \mathrm{E}_{\text {Bulk }}\right)
$$

$E_{\text {Slab }}$ is the energy of the slab, $A$ is the surface area of one side of the slab, $N_{\text {Slab }}$ is the number of atoms in the slab and $E_{\text {Bulk }}$ is the energy per atom for the bulk system. The factor of 2 accounts for the two surfaces of each slab, and the bulk energy $E_{\text {Bulk }}$ was calculated under the same amount of strain as the surface energy.

The functions presented in fig. 2 all demonstrate asymmetry about a vertical line centred at their minimum, and this behaviour was also seen in both their ESlab and E $_{\text {Bulk }}$ components. This asymmetry arises because $\sigma$ is purely in-plane. The work done by $\sigma$ causes a responsive outof-plane movement of the atoms which tends to shift the minimum of the surface energy curve away from the perhaps more intuitive $\sigma=0$ line. The asymmetry indicates that the simplest functional form required to fit the functions must be at least a cubic polynomial.

For each surface in fig. 2 two sets of data points are presented. The first are the discrete data points which were obtained from the density functional theory (DFT) simulations. The second is a smooth curve which is the weighted difference of two cubic polynomials. The first of these polynomials was fitted to the curve of Eslab versus $\sigma$ and was defined by eqn. (3):

$$
\mathrm{E}_{\text {Slab }}=\sum_{\mathrm{k}=0}^{3} \mathrm{C}_{\mathrm{k} ; \mathrm{Slab}} \sigma^{\mathrm{k}}
$$

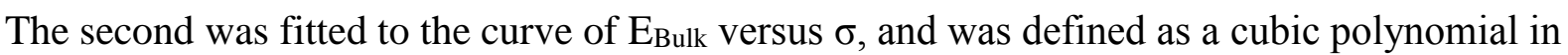
terms of $\sigma$ in a similar way to the definition of Eslab in eqn. (3). Their weighted difference was calculated according to eqn. (2) and is shown in fig. 2 as the set of smooth curves. The coefficients $C_{k ; \text { Slab }}$ are the linear and higher-order elastic constants of the material [19].

A normalised slab energy ESlab;Norm $_{\text {was defined as }}$

$$
\mathrm{E}_{\text {Slab;Norm }}=\left[1-\left(\frac{\mathrm{E}_{\text {Slab }}}{\mathrm{E}_{\text {Slab;0 }}}\right)\right] \times 100 \%
$$




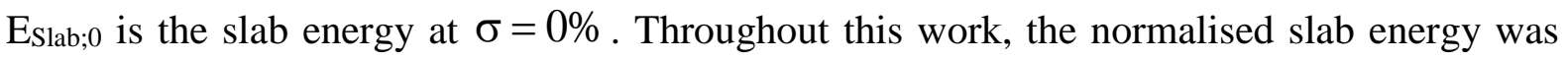
numerically treated as a percentage. The normalised slab energy ESlab;Norm was then modelled using a cubic polynomial

$$
\mathrm{E}_{\text {Slab;Norm }}=\sum_{\mathrm{k}=1}^{3} \mathrm{C}_{\mathrm{k}} \sigma^{\mathrm{k}}
$$

The utility of using $\mathrm{E}_{\text {Slab;Norm }}$ is that the polynomial expression on the right hand side of eqn. (5) does not contain a constant term. This reduces the search space required by eqn. (5) to 3 variables with $E_{S l a b ; 0}$, which is known from the DFT calculations, effectively removing $\mathrm{C}_{0}$. The $C_{k}$ are physically elastic constants of the material [19] in the same way as the $C_{k ; \text { Slab }}$ though they are now normalised.

Fig. 3 shows the $C_{k}$ for a range of transition metal surfaces. The first and second order coefficients $C_{1}$ and $C_{2}$ are positive whereas the third-order coefficient $C_{3}$ are generally negative, with the exceptions of $\mathrm{Ru}$ and $\mathrm{Os} . \mathrm{C}_{3}<0$ indicates that the strain energy will increase under stress due to an increase in the vibrational frequencies. This behaviour has also been seen recently on cadmium sulphide [25]. The abscissa of fig. 3 is $\left(\mathrm{dE}_{\mathrm{C}} / \mathrm{d} \sigma\right)$ where $\mathrm{E}_{\mathrm{C}}$ is the centre of the occupied d-band and was defined by

$E_{C}=\frac{\int_{-\infty}^{E_{F}}\left(E-E_{F}\right) n(E) d E}{\int_{-\infty}^{E_{F}} n(E) d E}$

$E_{F}$ is the Fermi energy and $n(E)$ is the density of states. For the results presented in fig. $3 E_{C}$ was evaluated for the surface atoms only. The reason for developing this formulation was because the documented importance of the occupied d-band centre in describing transition metal surfaces; this importance was outlined in the Introduction section at the start of this paper. Preliminary investigations in the current work showed that the surface atom occupied d-band centre $\mathrm{E}_{\mathrm{C}}$ generally shifts towards (away from) $\mathrm{E}_{\mathrm{F}}$ as the strain $\sigma$ became increasingly tensile (compressive). These changes were accompanied by a general decrease (increase) in the work function $\varphi$ of the surface of between 0.2 to $0.4 \mathrm{eV}$ for the range of $\sigma$ in the current work.

These observations strongly suggested the use of $\mathrm{E}_{\mathrm{C}}$ as an effective parameter; however, the current work is focussed on the response of these surfaces to changes in $\sigma$. Under these conditions $E_{C}$ was seen to change linearly with $\sigma$. The parameterisation was therefore possible in terms of the zero strain $(\sigma=0 \%)$ value of $E_{C}$ or of the slope $\left(\mathrm{dE}_{\mathrm{C}} / \mathrm{d} \sigma\right)$ and straightforward testing revealed that the strongest correlation existed between the $C_{k}$ and $\left(\mathrm{dE}_{\mathrm{C}} / \mathrm{d} \sigma\right)$ rather than between the $\mathrm{C}_{\mathrm{k}}$ and $\mathrm{E}_{\mathrm{C}}$.

Fig. 3 shows a strong geometrical dependence. This is evidenced by following the 'guides-tothe-eye' for both the (a) hexagonal, and (b) square surfaces. For the hexagonal graphs in (a) 
two types of surfaces are considered: FCC (111) and the HCP(0001). The difference between these two surfaces has been shown in fig. 1. The FCC surface has ABCABC layering whereas the HCP surface has ABABAB layering. This difference in the third layer causes two distinctly different curves to appear on each of the panels in fig. 3 (a). The lower relative importance of the electronic state of the surface is demonstrated by the closeness of surfaces of either FCC $(111)$ or $\mathrm{HCP}(0001)$ to one of the solid curves, independent of their electronic state. The solid lines are not intended for precise statistical analysis and consequently are presented as guides. Even with this simple presentation he different correlations for the FCC and HCP surfaces is clear. These conclusions are support by the results in fig. 3 (b) where no geometrical differences exist between the different surfaces except the lattice parameter, and electronic differences exist between the different surfaces. In these figures a single correlation only is seen.

In fig. 3 only the surface layer atoms were used to evaluate $E_{C}$. Fig. 4 shows the different values of $\left(\mathrm{dE}_{\mathrm{C}} / \mathrm{d} \sigma\right)$ that are obtained by evaluating $\mathrm{E}_{\mathrm{C}}$ across successive subsurface layers. The data show the range of $\left(\mathrm{dE}_{\mathrm{C}} / \mathrm{d} \sigma\right)$ for each system and demonstrate the tolerance of the approximation made in the fig. 3 to only select the surface atom contribution to $\left(\mathrm{dE}_{\mathrm{C}} / \mathrm{d} \sigma\right)$. The large dispersion of $\left(\mathrm{dE}_{\mathrm{C}} / \mathrm{d \sigma}\right)$ for individual systems shown in fig. 4 is sufficient to degrade the correlation shown in fig. 3. However, more correlated behaviour is seen in the surface relaxation behaviour shown in fig. 5. This figure shows the rate of change of surface relaxation $\left(\mathrm{dz}_{\mathrm{L}} / \mathrm{d} \sigma\right)$. The parameter is negative for all systems indicating that the surface and selvedge atoms move towards (away from) the bulk as $\sigma$ becomes increasingly tensile (compressive). This behaviour is seen even for Pd which showed the well-documented an expansion in the surface layer at $\sigma=0 \%$. The $\left(\mathrm{dz}_{\mathrm{L}} / \mathrm{d} \sigma\right)$ show larger values for atomic layers at the surface and progressively reduce as the selvedge layer is traversed towards the bulk. This periodic behaviour in the geometric nature of these surfaces under strain compares sharply with the less periodic layer-resolved behaviour seen in fig. 4 for the occupied d-band centre, and together with the structure-dependent correlation seen in fig. 3 identifies that, under strain, the geometry of these surface system rather than their electronic nature controls their surface energy and nonelastic response.

\section{Conclusions}

The behaviour under strain of the surface energies of the BCC Fe(100), HCP Co(0001), $\mathrm{Ru}(0001)$ and $\mathrm{Os}(0001)$, and the (111) and (100) surfaces of FCC Ni, Cu, Rh, Pd, Ag, Ir, Pt, and $\mathrm{Au}$ have been investigated using a combination of density functional theory and the formalism on non-linear elasticity theory.

The surface energies were accurately modelled using cubic polynomials where the variable space is strain $\sigma$. The coefficients in these polynomials demonstrate strongly geometrydependent behaviour as distinct correlations have been seen between to coefficients for the $\mathrm{FCC}(111), \mathrm{HCP}(0001)$ and the $\mathrm{FCC}(100)$ and $\mathrm{BCC}(100)$ surfaces, irrespective of their electronic nature. 
These correlations have been identified by use of a strain dependent variable, the layer-resolved slope $\left(\mathrm{dE}_{\mathrm{C}} / \mathrm{d} \sigma\right)$ where $E_{C}$ is the centre of the occupied d-band. The layer-resolution of this approximation has been investigated and a second set of common geometrically-based parameters, the $\left(\mathrm{dz}_{\mathrm{L}} / \mathrm{d} \sigma\right)$, have been identified. The commonality of the $\left(d z_{L} / d \sigma\right)$ between surfaces is shown again to be in contrast to the electronic behaviour of the layer-resolved $\left(\mathrm{dE}_{\mathrm{C}} / \mathrm{d} \sigma\right)$ and establishes the importance geometrical state of the surface in understanding the behaviour of surface energy and non-linear elastic constants of these systems when they are under strain.

\section{Acknowledgements}

This work was supported by the author's membership of the UK's HEC Materials Chemistry Consortium, which is funded by the EPSRC (EP/L000202) and used the ARCHER UK National Supercomputing Service (http://www.archer.ac.uk).

\section{References}

[1] P. Müller, and A. Saùl. Surf. Sci. Reps. 54 (2004) 157-258.

[2] W. Haiss. Rep. Prog. Phys. 64 (2001) 591-648.

[3] M. Blanco-Rey, and S. J. Jenkins. J. Phys.: Condens. Matter. 22 (2010) 135007.

[4] Y. Shiihara, and M. Kohyama. Surf. Sci. 644 (2016) 122-128.

[5] D. Pettifor. J. Phys. F: Met. Phys. 7 (1977) 1009.

[6] Y. Shiihara, M. Kohyama, and S. Ishibashi. Phys. Rev. B: Condens. Matter 87 (2013) 125430.

[7] I. G. Shuttleworth. Appl. Surf. Sci. 378 (2016) 286-292.

[8] R. Johansson, R. Ahuja, O. Eriksson, B. Hjörvarsson, and R. H. Scheicher.

[9] L. Vitos, A. V. Ruban, H. L. Skriver, and J. Kollár. Surf. Sci. 411 (1998) 186-202.

[10] H. L. Skriver, and N. M. Rosengaard. Phys. Rev. B: Condens. Matter 46 (1992) 7157.

[11] A. M. Rodriguez, G. Bozzolo, and J. Ferrante. Surf. Sci. 289 (1993) 100-126.

[12] M. Methfessel, D. Henning, and M. Scheffler. Phys. Rev. B: Condens. Matter 46 (1992) 4816.

[13] J. Kollár, L. Vitos, and H. L. Skriver. Phys. Rev. B: Condens. Matter 46 (1992) 11288.

[14] A. Kiejna, and V. V. Pogosov. Phys. Rev. B: Condens. Matter 62 (2000) 10445.

[15] D.-P. Ji, Q. Zhu, and S.-Q. Wang. Surf. Sci. 651 (2016) 137-146. 
[16] N. E. Singh-Miller, and N. Marzari.

Phys. Rev. B: Condens. Matter 80 (2009) 1235407.

[17] S. D. Wang, and W. Li. Physica B 406 (2011) 4046-4051.

[18] D. I. Bazhanov, I. V. Mutigullin, A. A. Knizhnik, B. V. Potapkin, A. A. Bagaturyants, L. R. C. Fonseca, and M. W. Stoker. J. Appl. Phys. 107 (2010) 083521.

[19] Y. Hiki. Annu. Rev. Mater. Sci. 11 (1981) 51-73.

[20] C. Wang, J. Gu, W. Zhang, B. Sun, D. Liu, and G. Liu. Comput. Mater. Sci. 124 (2016) 375-383.

[21] P. Giannozzi, S. Baroni, N. Bonini, M. Calandra, R. Car, C. Cavazzoni, D. Ceresoli, G.L. Chiarotti, M. Cococcioni, I. Dabo, A. Dal Corso, S. Fabris, G. Fratesi, S. deGironcoli, R. Gebauer, U. Gerstmann, C. Gougoussis, A. Kokalj, M. Lazzeri, L.Martin-Samos, N. Marzari, F. Mauri, R. Mazzarello, S. Paolini, A. Pasquarello, L.Paulatto, C. Sbraccia, S. Scandolo, G. Sclauzero, A.P. Seitsonen, A. Smogunov, P.Umari, and R.M. Wentzcovitch. J. Phys. Condens. Matter. 21 (2009) 395502.

[22] M. Methfessel, and A.T. Paxton.

Phys. Rev. B: Condens. Matter 40 (1989) 3616-3621.

[23] The Fe.pbe-spn-rrkjus_psl.0.2.1.UPF, x.pbe-nd-rrkjus.UPF, where $\mathrm{x}=\mathrm{Co}$ or Ni, y.pbe-mt fhi.UPF, where $\mathrm{y}=\mathrm{Cu}, \mathrm{Ru}, \mathrm{Rh}, \mathrm{Pd}, \mathrm{Os}$, Ir or Pt, and z.pbesol-dnrrkjus_psl.0.1.UPF, where $\mathrm{y}=\mathrm{Ag}$ or $\mathrm{Au}$, pseudopotentials were used from the Quantum ESPRESSO pseudopotential data base: http://www.quantumespresso.org/pseudopotentials.

[24] A. Dal Corso. Comput. Mater. Sci. 95 (2014) 337-350.

[25] S. Jones, and S. Menon. Eur. Phys. J. B 87(4) (2014) 85. 
Table 1. Summary of the equilibrium bulk lattice constants determined computationally in the current work ( $\mathrm{a}_{\text {theo }}$ ) and experimentally ( $\mathrm{a}_{\text {expt }}$ ). All dimensions are in $\AA$.

\begin{tabular}{|l|l|l|}
\hline \multicolumn{1}{|c|}{ Element } & \multicolumn{1}{|c|}{ atheo } & \multicolumn{1}{c|}{$\mathrm{a}_{\text {expt }}$} \\
\hline $\mathrm{Fe}$ & 2.838 & 2.867 \\
\hline $\mathrm{Co}$ & 2.491 & 2.507 \\
\hline $\mathrm{Ni}$ & 3.518 & 3.524 \\
\hline $\mathrm{Cu}$ & 3.680 & 3.615 \\
\hline $\mathrm{Ru}$ & 2.698 & 2.706 \\
\hline $\mathrm{Rh}$ & 3.850 & 3.803 \\
\hline $\mathrm{Pd}$ & 3.962 & 3.891 \\
\hline $\mathrm{Ag}$ & 4.073 & 4.085 \\
\hline $\mathrm{Os}$ & 2.745 & 2.734 \\
\hline $\mathrm{Ir}$ & 3.898 & 3.839 \\
\hline $\mathrm{Pt}$ & 3.981 & 3.924 \\
\hline $\mathrm{Au}$ & 4.073 & 4.078 \\
\hline
\end{tabular}


Fig. 1. Schematic showing (a) hexagonal FCC (111), (b) hexagonal HCP (0001), and (c) square FCC (100) and BCC (100) surfaces. Surface (second/third) layer metal atoms are shown by white (light grey/dark grey) circles, respectively. $\mathbf{a}_{1}$ and $\mathbf{a} 2$ are the primitive surface vectors for each system, and are $(2 \times 2)$ for clarity. The dashed horizontal lines in the cross-sectional views show the ideal, bulk terminated plane heights $Z_{L}$,ideal where the subscript ' $L$ ' denotes 'Surf', '2', or ' 3 , and the solid lines show the actual plane heights $\mathrm{Z}_{\mathrm{L}}$.

(a)
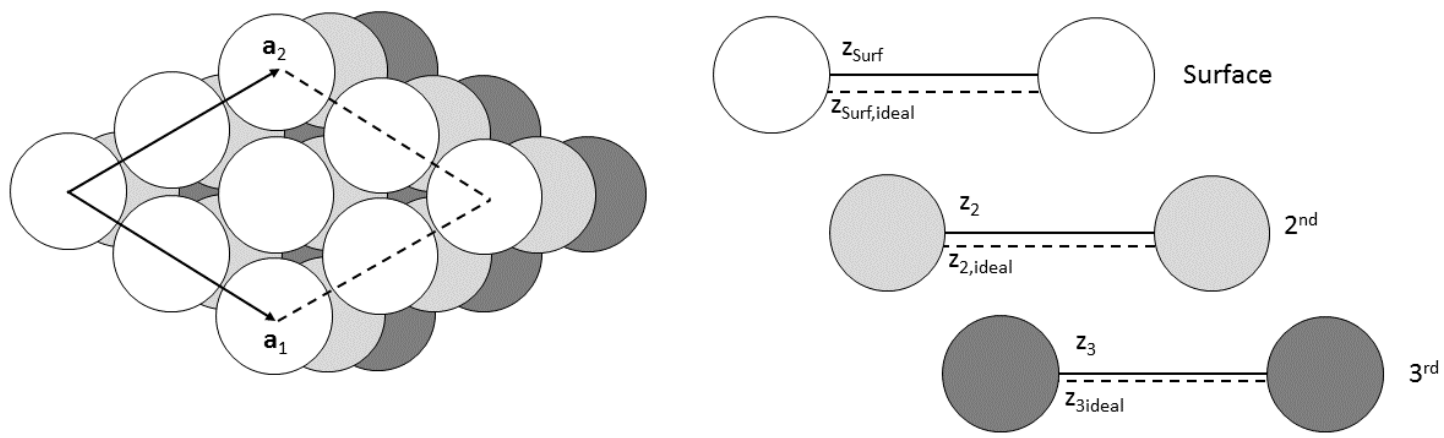

(b)
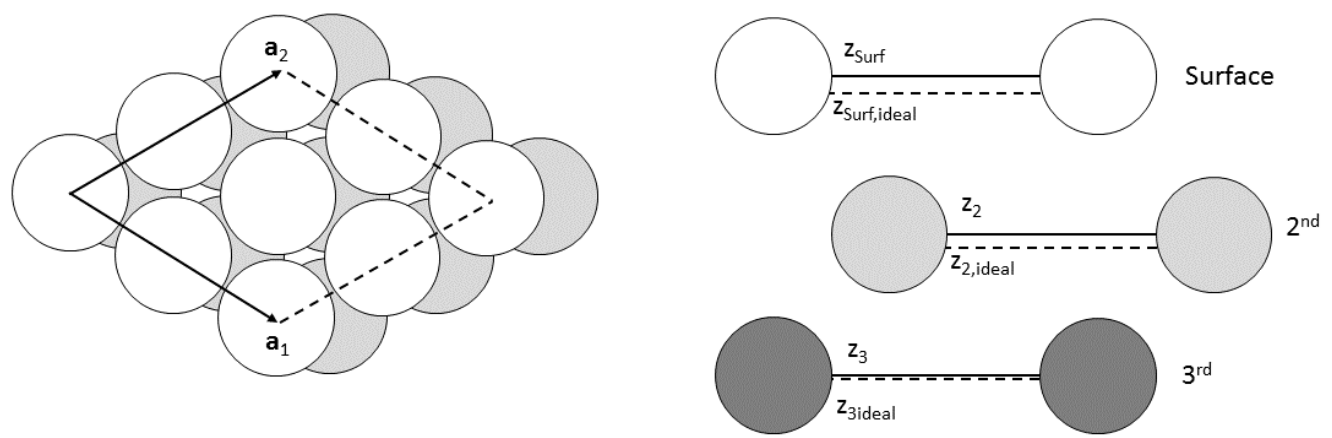

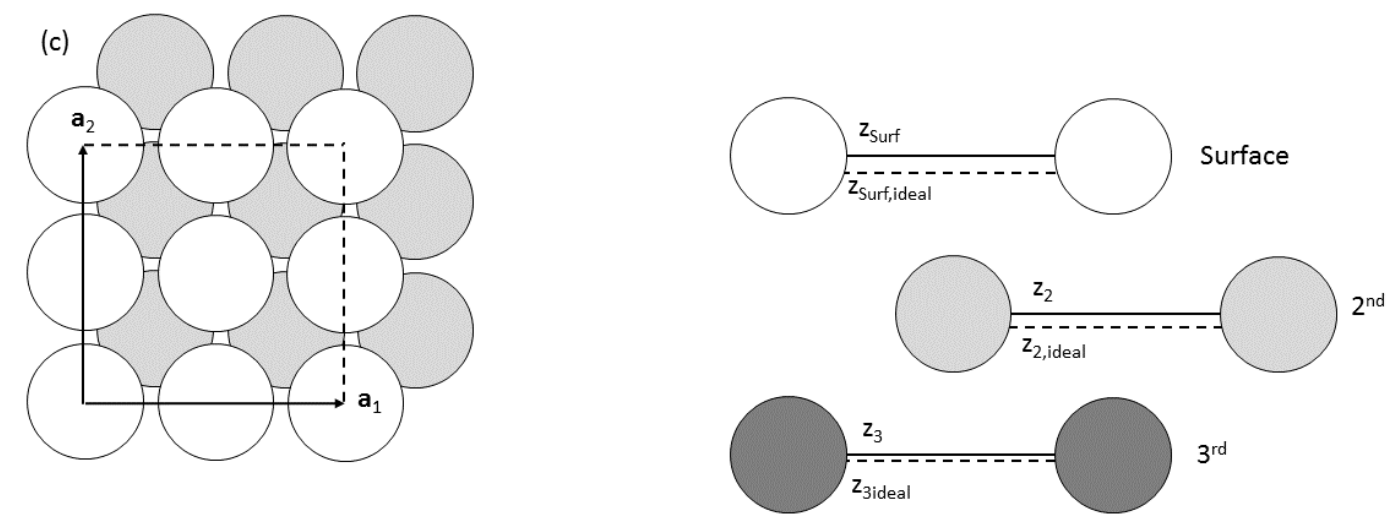
Fig. 2. Surface energy $E_{S}$ for metallic (a-f) FCC (111) and (100), (g) HCP (0001) and (h) BCC (100) surfaces.
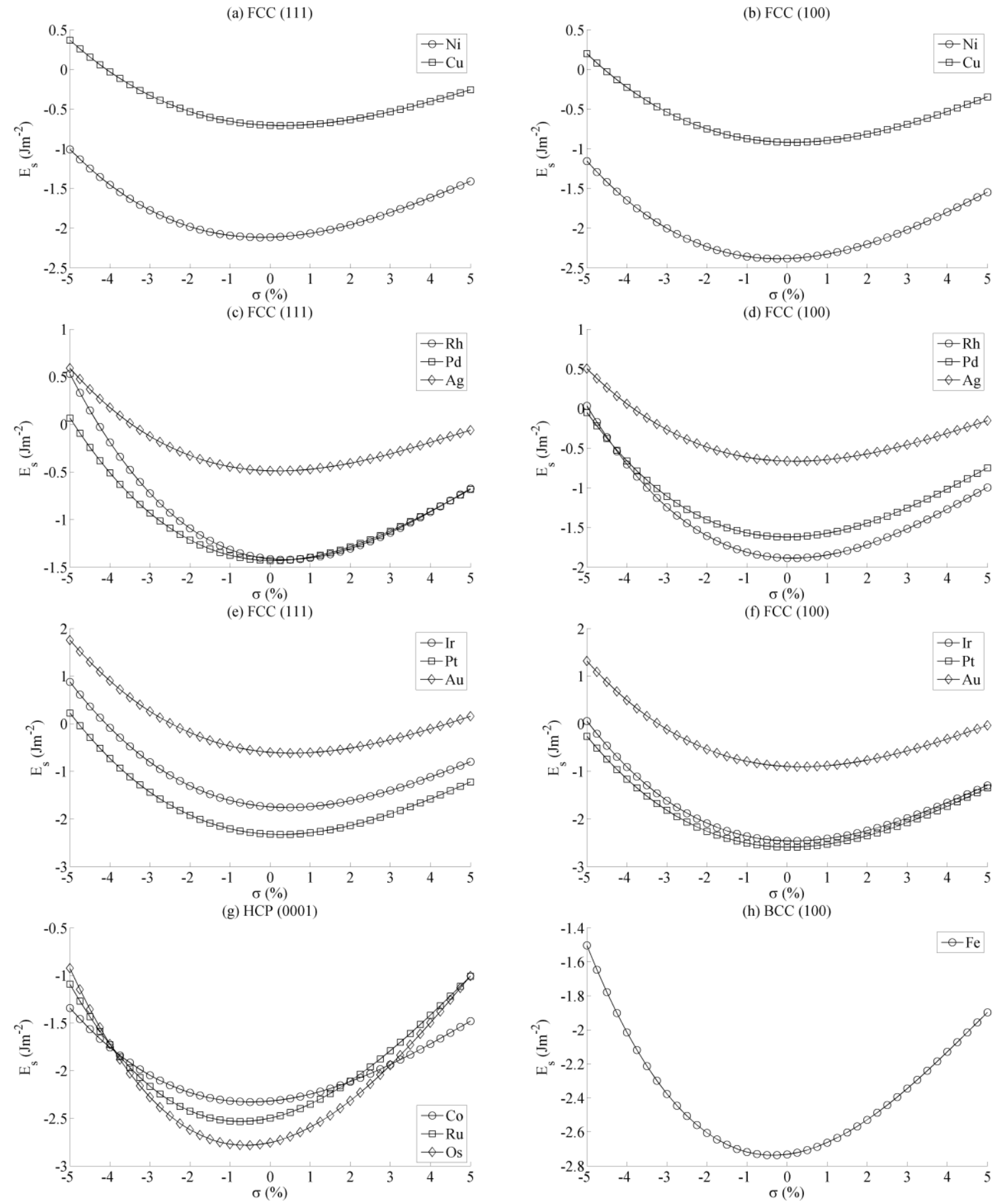
Fig. 3. Normalised slab energy $E_{S l a b ; N o r m}$ coefficients $C_{1}, \ldots, C_{3}$ versus the rate of change of occupied d-band centre with strain $\mathrm{dE}_{\mathrm{c}} / \mathrm{d} \sigma$, for (a) hexagonal FCC(111) and $\mathrm{HCP}(0001)$, and (b) square FCC(100) and BCC (100) surfaces. The occupied d-band centres $E_{C}$ were calculated for the surface atoms only. The solid lines are a guide to the eye.

(a)
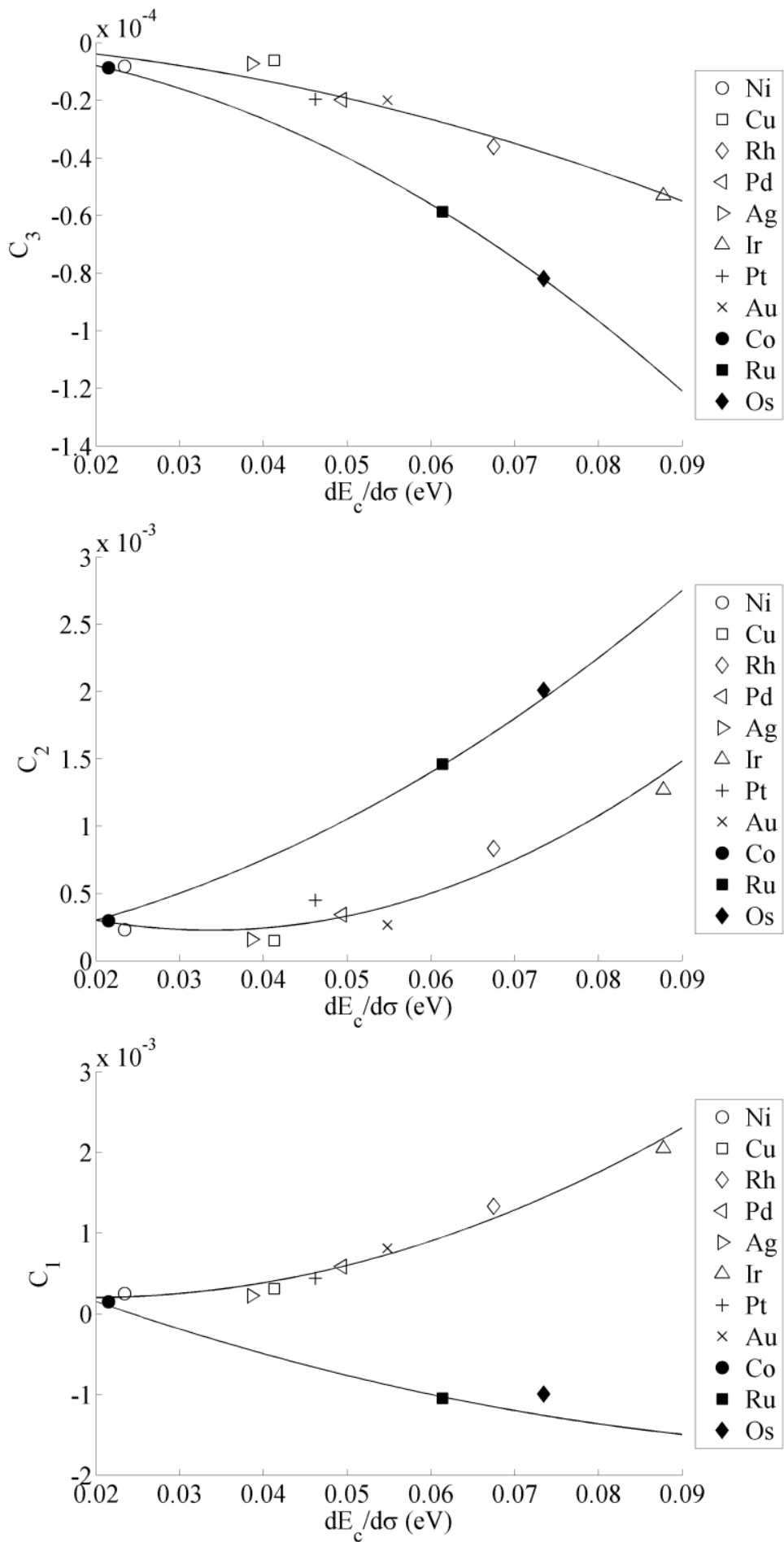
(b)
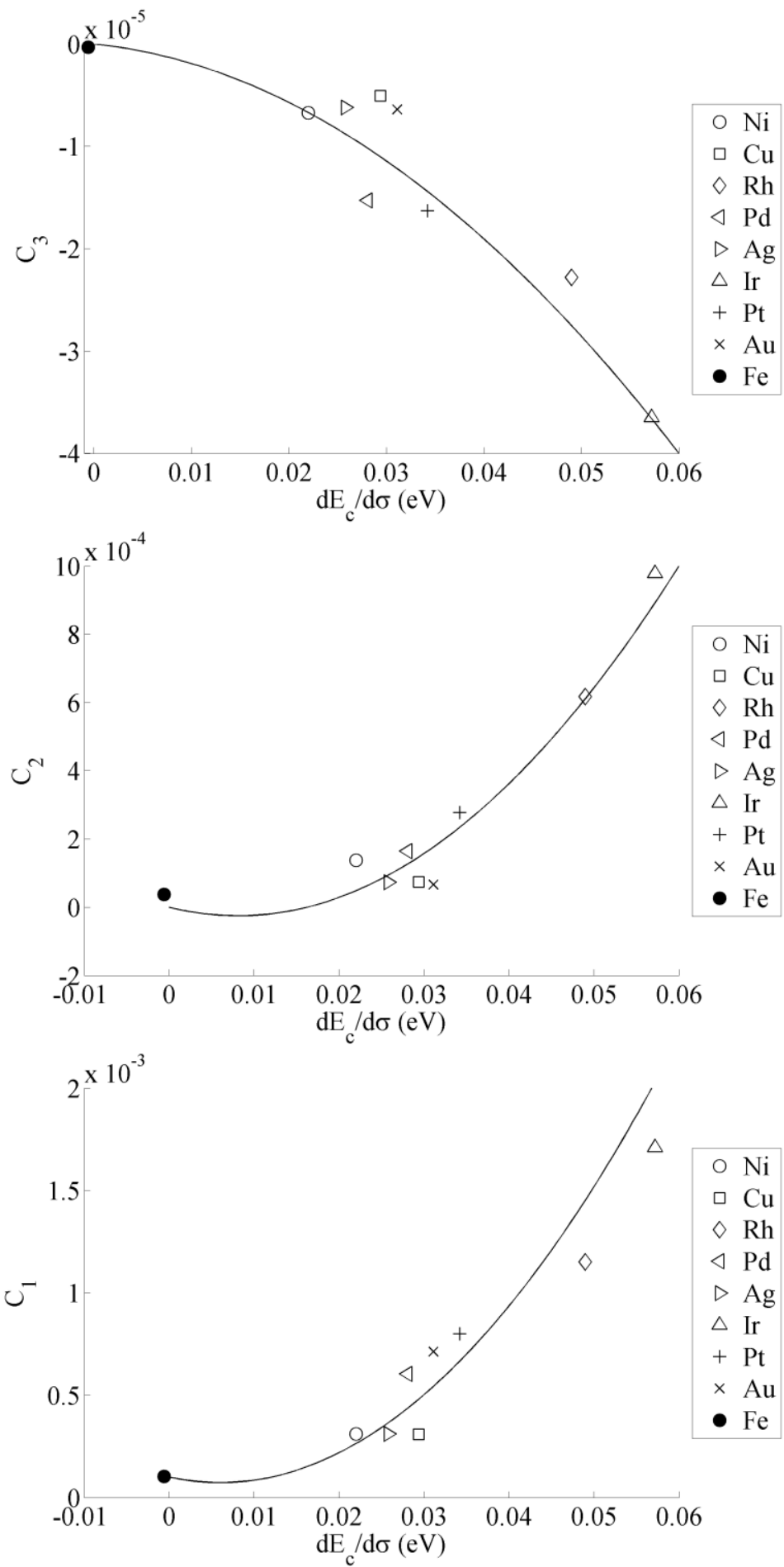
Fig. 4. Layer-resolved rate of change of the occupied d-band centre with strain $\mathrm{dE}_{\mathrm{c}} / \mathrm{d} \sigma$ for (a) hexagonal, and (b) square, surfaces. For each surface, 4 data points are shown; the leftmost is $\mathrm{dE}_{\mathrm{c}} / \mathrm{d} \sigma$ for the surface layer metal atoms, the second leftmost is $\mathrm{dE}_{\mathrm{c}} / \mathrm{d} \sigma$ for the second layer atoms, and so on.

(a)

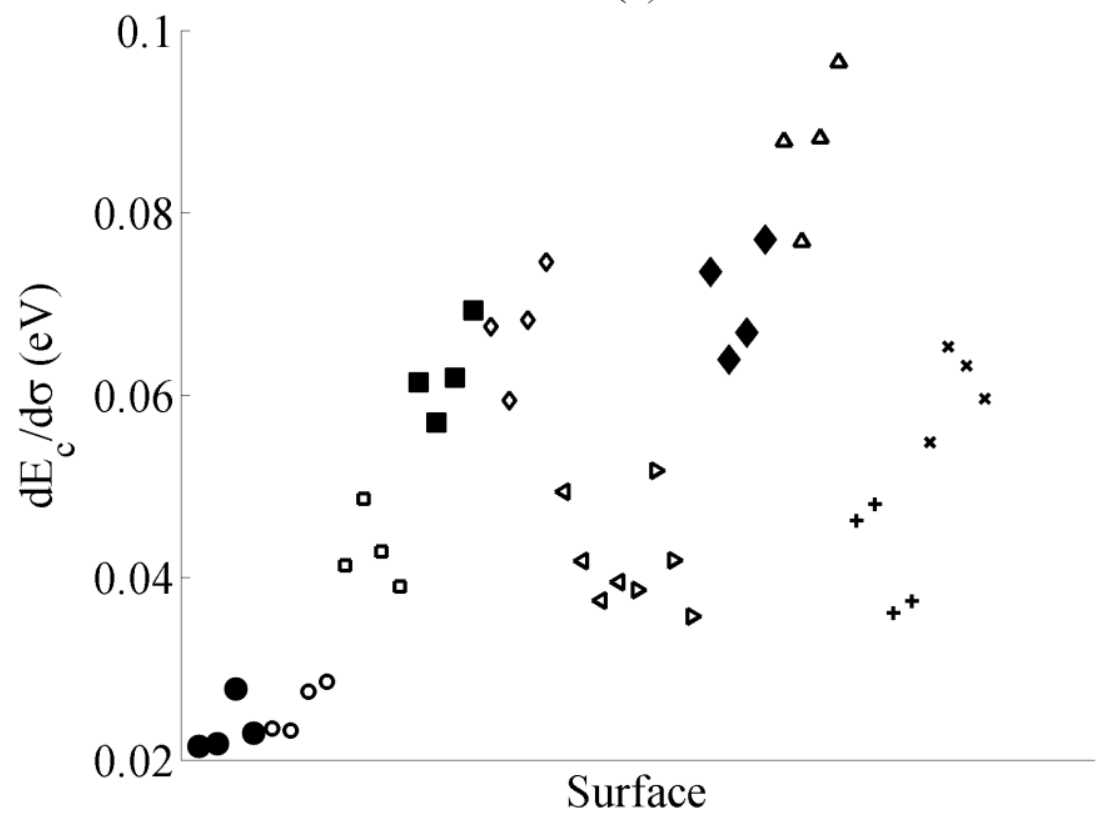

- $\operatorname{Co}(0001)$

- Ni(111)

- $\mathrm{Cu}(111)$

- $\mathrm{Ru}(0001)$

- Rh(111)

$\triangleleft \operatorname{Pd}(111)$

$\triangleright \operatorname{Ag}(111)$

- Os(0001)

$\Delta \operatorname{Ir}(111)$

$+\operatorname{Pt}(111)$

$\times \mathrm{Au}(111)$

(b)

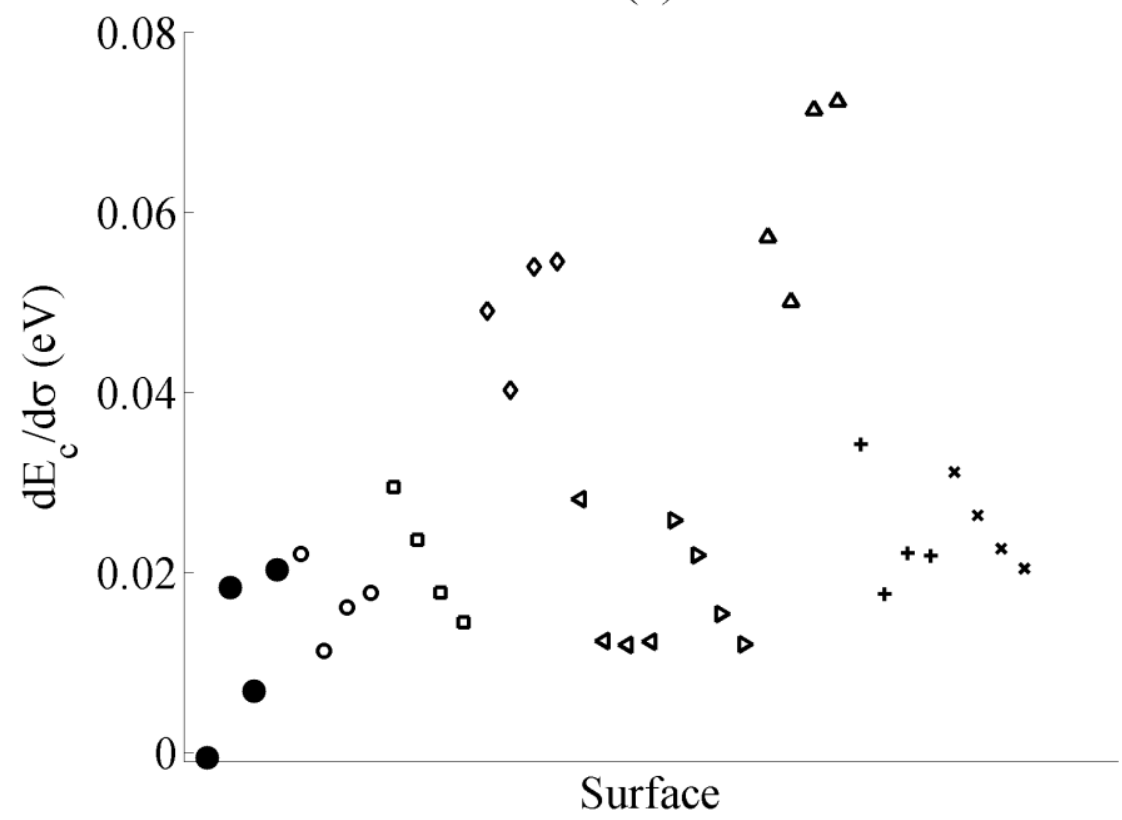

- $\mathrm{Fe}(100)$

- $\mathrm{Ni}(100)$

- $\mathrm{Cu}(100)$

- Rh(100)

$\triangleleft \operatorname{Pd}(100)$

$\triangleright \operatorname{Ag}(100)$

$\Delta \operatorname{Ir}(100)$

+ $\operatorname{Pt}(100)$

$\times \mathrm{Au}(100)$ 
Fig. 5. Rate of change of the normalised layer spacing $\mathrm{z}_{\mathrm{NL}}$ with strain. The normalised layer spacing $\mathrm{Z}_{\mathrm{NL}}$ is the layer spacing $\mathrm{Z}_{\mathrm{L}}$ defined in fig. 1 normalised to the bulk lattice constant.

(a) FCC (111)
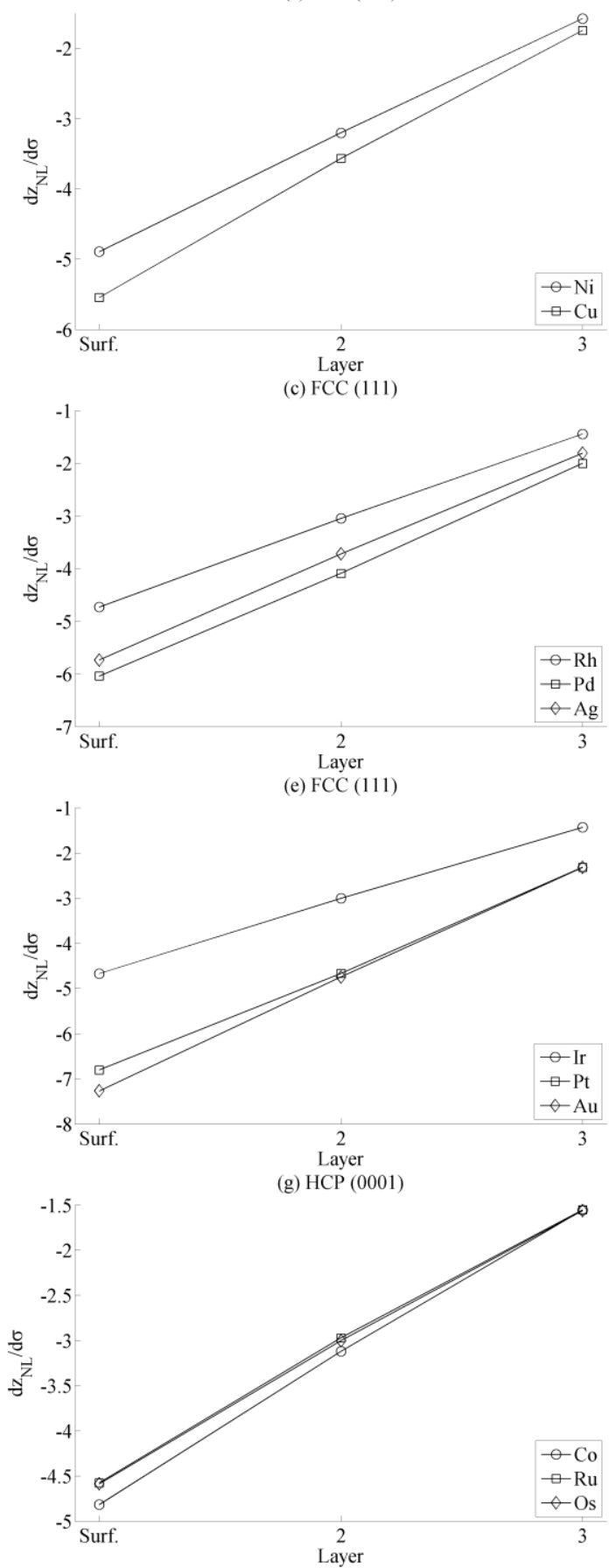

(b) FCC (100)
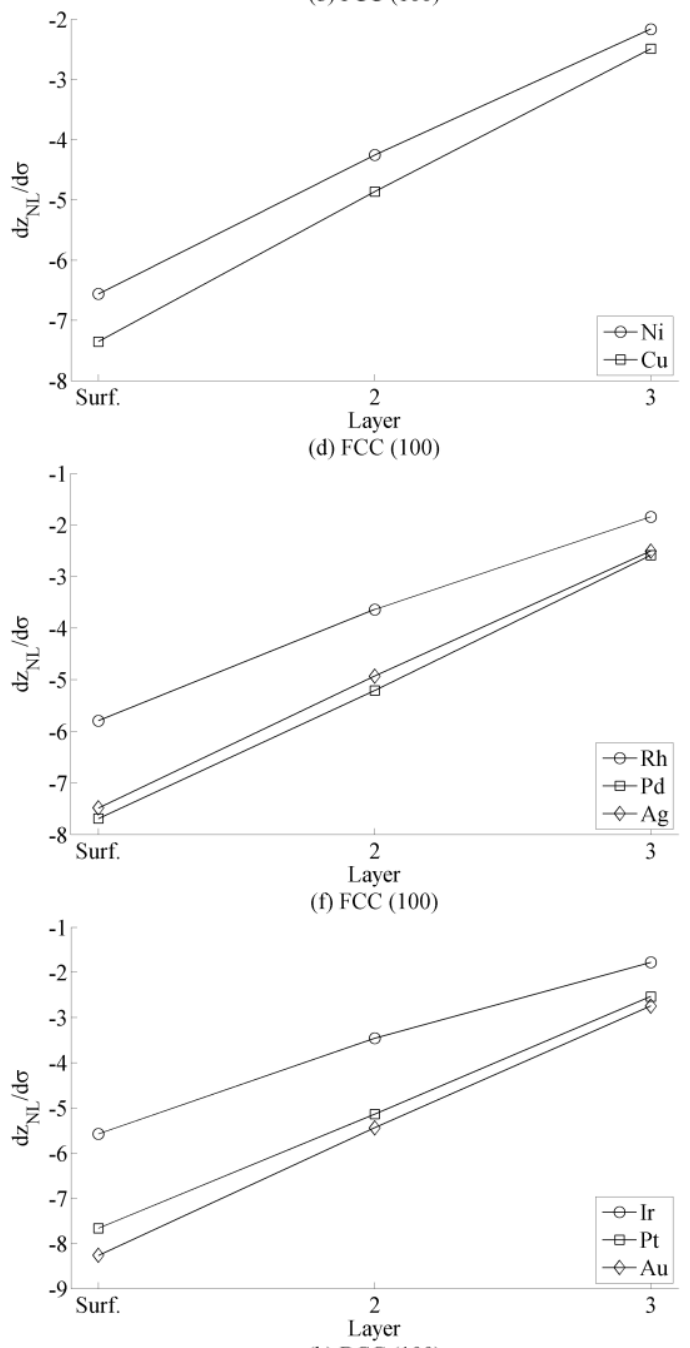

(h) BCC (100)

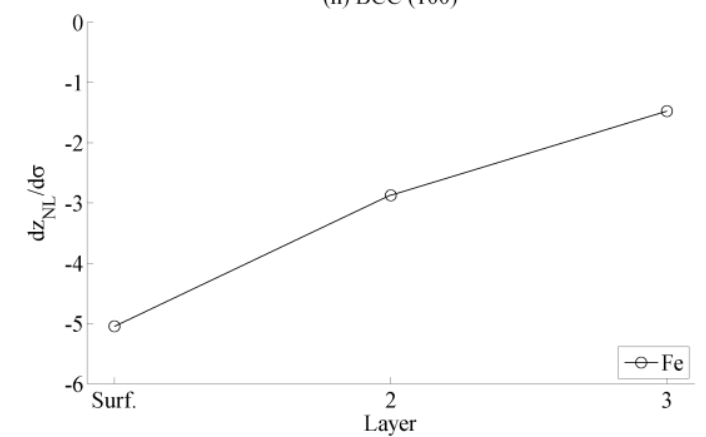

